\title{
La Dopant Segregation at Threading Dislocations in La:BaSnO3 Thin Films
}

Hwanhui Yun ${ }^{1}$, Abhinav Prakash ${ }^{2}$, Bharat Jalan ${ }^{1}$ and K. Andre Mkhoyan ${ }^{1}$

${ }^{1}$ University of Minnesota-Twin Cities, Minneapolis, Minnesota, United States, ${ }^{2}$ Argonne National Laboratory, Lemont, Illinois, United States

Threading dislocation (TD) is the one of the most commonly observed crystalline defects in perovskite oxide thin films. ${ }^{1}$ TDs in thin films can change the local atomic and electronic structures and considerably modify its properties. For instance, TDs in $\mathrm{BaSnO}_{3}$, one of the promising perovskite oxides due to the high room temperature mobility and optical transparency, are known to be the major defects limiting the electronic transport of the material by reducing the carrier mobility. ${ }^{2-4}$ However, relatively little research has been done on the local atomic and electronic structures of $\mathrm{TDs}$ in $\mathrm{BaSnO}_{3}$. Here compositional and electronic structures of TDs in La-doped $\mathrm{BaSnO}_{3}$ thin films are explored by using analytical STEM.

La-doped $\mathrm{BaSnO}_{3}$ films studied here were grown on $\mathrm{SrTiO}_{3}$ and $\mathrm{LaAlO}_{3}$ substrates by hybrid molecular beam epitaxy. ${ }^{5}$ Plan-view STEM samples were prepared by mechanical polishing. STEM experiments were carried out using aberration-corrected FEI Titan G2 60-300 (S)TEM equipped with a CEOS DCOR probe corrector, Schottky extreme field emission gun, and a monochromator. STEM-EDX and STEMEELS data were acquired using the FEI Super-X EDX detector and Gatan Enfinium ER EELS.

Figure 1a shows plan-view HAADF- and LAADF-STEM images of La-doped BaSnO3. Two different types of TDs, single TD $(\mathbf{b}=(100))$ and dissociated TD $(\mathbf{b}=(110))$, are shown as bright- single spot and paired spots in the LAADF-STEM image. High resolution HAADF-STEM images show the atomic configuration of the TDs and are used to obtain the strain field map around the TDs via geometric phase analysis (Figure 1b). ${ }^{6}$ Atomic-resolution EDX elemental maps are acquired from the TDs revealing that La dopants are segregated at specific regions (Figure 2), and correlation between the preferential La segregation and the strain field around the TDs is observed. The effects of La segregation on TDs are further investigated by comparative analysis of undoped- and La-doped $\mathrm{BaSnO}_{3}$ thin films, where STEMEELS analysis evidences the modulation of the electronic structures originated from the segregated dopants. 5,7 

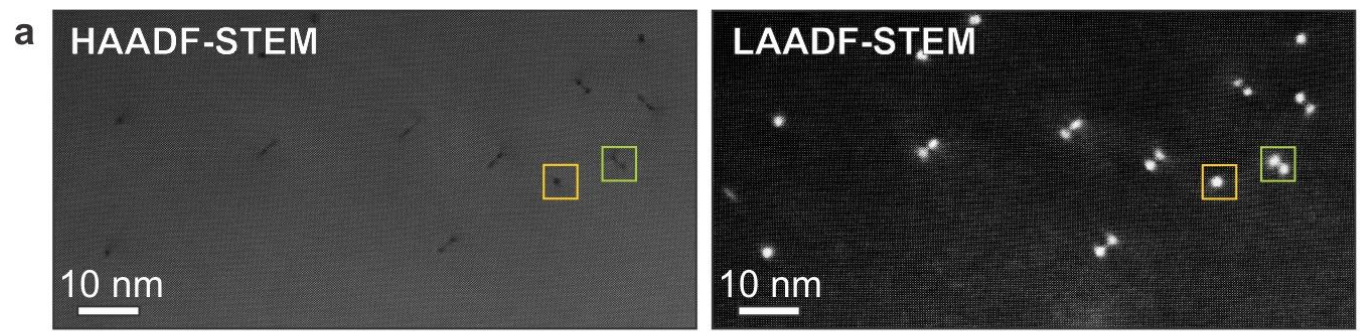

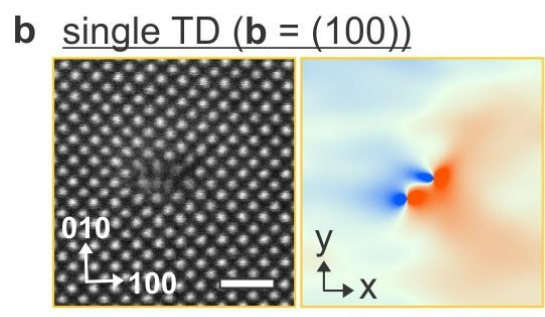

HAADF

dissociated TD $(\mathbf{b}=(110))$

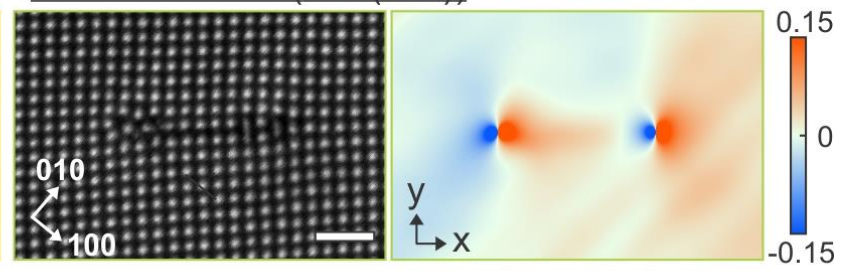

HAADF

Strain $\left(\varepsilon_{x x}\right)$

Figure 1. (a) Plan-view HAADF- and LAADF-STEM images of a La:BaSnO3 thin film. One of singleand dissociated TDs are highlighted in yellow and green boxes, respectively. (b) Strain field map around TDs. Color code of strain map: compressive strain-blue, tensile strain-red. Scale bars are $1 \mathrm{~nm}$.

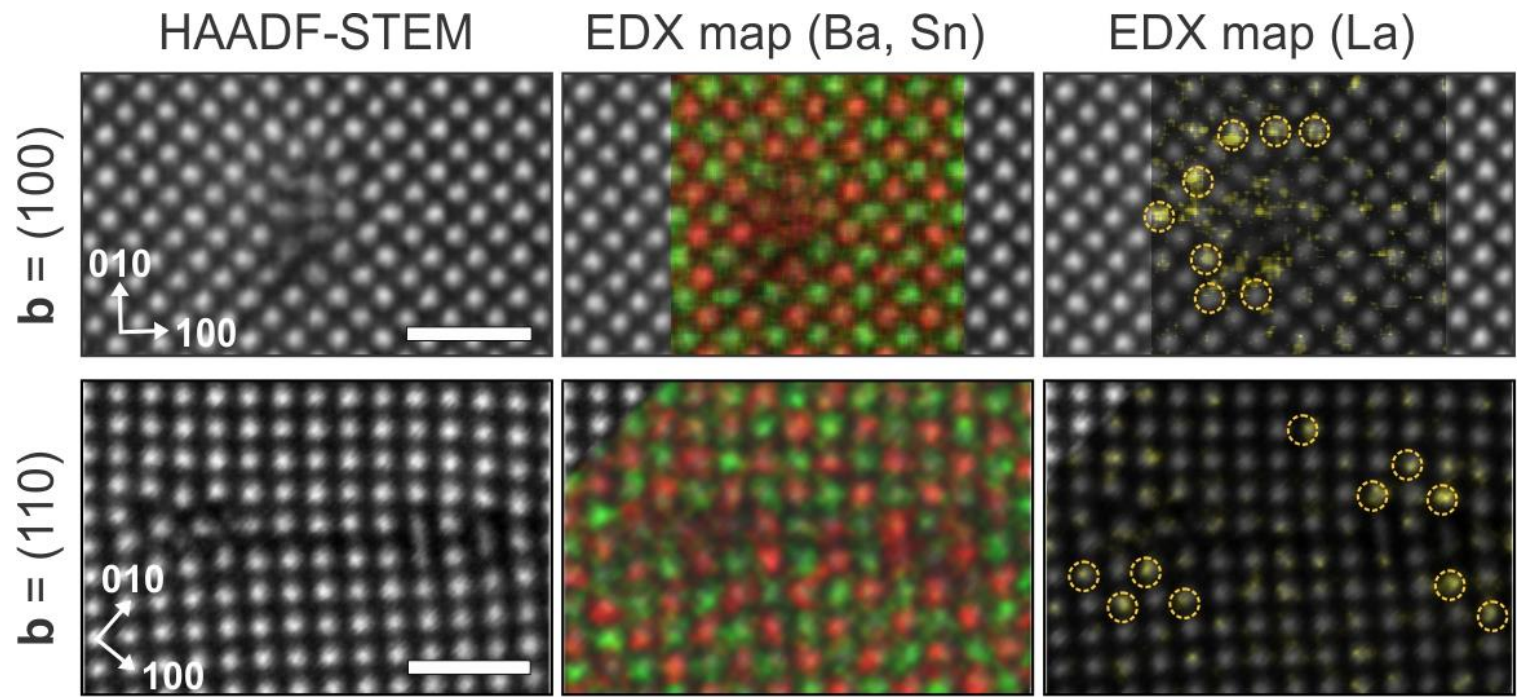

Figure 2. Atomic-resolution EDX elemental map of TDs in $\mathrm{La}: \mathrm{BaSnO} 3 . \mathrm{K} \alpha$ and $\mathrm{K} \beta \mathrm{x}$-ray emission signals were used for each elemental map. The EDX maps are overlapped on the HAADF-STEM image. Discernable La segregation sites are marked with circles. Color code: Ba-green, Sn-red, La-yellow. Scale bars are $1 \mathrm{~nm}$.

References

1. D. Hull et al.,"Introduction to Dislocations", fifth edition, (Butterworth-Heinemann, Oxford)

2. X. Luo et al., Appl. Phys. Lett. 2012, 100, (17), 172112.

3. H. J. Kim et al., Appl. Phys. Express 2012, 5, (6), 061102.

4. A. Prakash et al., Nat. Comm. 2017, 8, (1), 15167.

5. A. Prakash et al., J. Vac. Sci. Technol. A 2015, 33, (6), 060608.

6. M. J. Hÿtch et al., Ultramicroscopy 1998, 74, (3), 131-146.

7. The project was partially supported by UMN MRSEC program DMR-1420013. Film growth and characterization was primarily supported by the U.S. DOE BES through the Grant DE-SC-0020211. 